\title{
Predictive Control of Induction Motor Drive Using dSPACE Platform
}

\author{
Pavel Karlovský and Jiří Lettl \\ Department of Electric Drives and Traction, Faculty of Electrical Engineering, \\ Czech Technical University in Prague, Prague, Czech Republic \\ email:karlopav@fel.cvut.cz,lettl@fel.cvut.cz
}

\begin{abstract}
Recently, the induction motor drives have become very popular in the area of controlled drives. Nowadays, a great effort is put on increasing their efficiency. The most widely used control method is the direct torque control (DTC). However, this method suffers from the torque ripple and current waveform distortion. The modification using predictive algorithms is becoming a popular approach. This paper presents a predictive control on the dSPACE DS1103 system and compares the method with DTC.
\end{abstract}

Keywords - direct torque control, predictive control, induction motor drive

\section{INTRODUCTION}

In the field of electric drives, a drive with an induction motor is the most widely used. It is caused by its simplicity, robustness, and low price. The disadvantage is the fact, that in many applications this drive needs sophisticated control algorithms. The commonly used strategy is the direct torque control strategy. It was first presented in 1986 by Isao Takahashi and Toshihiko Noguchi [1]. The method is relatively easy to implement. However, one of the main disadvantages is caused by relatively high ripples in the torque waveform. That is because of the difficulties of maintaining the desired hysteresis. A lot of modifications have been performed since then. They are mainly the use of space vector modulation [2], dividing the flux vector space into more sectors [3], use of multilevel inverter [4] and other [5], [6], [7]. This paper deals with an attempt to enhance the DTC algorithm in a specific induction motor drive with a predictive method. This predictive method consists in the similar principles as the classical DTC algorithm. The prediction of the voltage vector enables the method to decrease the torque ripples at the same number of switching of the power transistors [5] and [6].

\section{PREDICTIVE METHOD}

\section{A. Figures and Tables - Subsection Example}

The predictive method is based on the same torque equation as the DTC method. The equation is a cross product of the stator and rotor magnetic flux vectors (1):

$$
T=\frac{L_{\mathrm{m}}}{L_{\Delta}} p_{\mathrm{p}} \cdot \psi_{\mathrm{s}} \times \psi_{\mathrm{r}}=\frac{L_{\mathrm{m}}}{L_{\Delta}} p_{\mathrm{p}} \cdot\left|\psi_{\mathrm{s}}\right| \cdot\left|\psi_{\mathrm{r}}\right| \cdot \sin \theta_{\mathrm{sr}}
$$

where $T$ is torque, $L_{\mathrm{m}}$ is mutual inductance, $L_{\mathrm{s}}$ is stator inductance, $L_{\mathrm{r}}$ is rotor inductance, $L_{\Delta}=L_{\mathrm{s}} \cdot L_{\mathrm{r}}-L_{\mathrm{m}}{ }^{2}, p_{\mathrm{p}}$ is number of pole pairs $\Psi_{\mathrm{r}}$ is rotor magnetic flux, $\Psi_{\mathrm{s}}$ is stator magnetic flux and $\theta_{\mathrm{sr}}$ is the angle between the stator and rotor flux vectors.
In electric drives, constant flux amplitude is often required. Then, the generated torque is dependent only on the angle between these flux vectors. The classical DTC does not know the exact value of the angle, but it is able to increase it or decrease it by applying the specific voltage vector. This is decided according to the hysteresis controllers that compare the actual torque and flux values with their references. Unlike the DTC method, in every step, the predictive method calculates not only the torque and stator flux vectors but moreover the rotor flux vector. Thanks to it, the predictive method knows the angle and therefore how would any voltage vector affect the torque waveform in the next step and choose the most suitable one. The working principle is depicted in Fig. 1. The algorithm consists of three parts. They are the induction motor model, predictive controller and block of evaluating results and choosing the right voltage vector.

The predictive method requires the knowledge of the stator and rotor flux vectors and torque generated by the motor. Because of that, the induction motor (IM) model is important for the method. It is calculated from the induction motor equivalent circuit. The measured variables are two phases of the current and voltage levels in the DC link. The voltage vector is then reconstructed from the voltage level in the DC link and known transistor combination set on the inverter. From these variables, the stator and rotor flux vectors as well as torque are calculated according to the equations (2), (3), (4):

$$
\begin{gathered}
\Psi_{\mathrm{s}}=\int\left(u_{\mathrm{s}}-R_{\mathrm{s}} \cdot i_{\mathrm{s}}\right) \mathrm{d} t \\
\Psi_{\mathrm{r}}=\left(\frac{\Psi_{\mathrm{s}}}{L_{\mathrm{m}}}-i_{\mathrm{s}}\right) \cdot L_{\mathrm{l}}+\Psi_{\mathrm{s}} \\
T=\frac{3}{2} \cdot p_{\mathrm{p}} \cdot\left(\Psi_{\alpha \mathrm{s}} \cdot i_{\beta \mathrm{s}}-\Psi_{\beta \mathrm{s}} \cdot i_{\alpha \mathrm{s}}\right)
\end{gathered}
$$

where $u_{\mathrm{s}}$ is stator voltage, $R_{\mathrm{s}}$ is stator resistance, $i_{\mathrm{s}}$ is stator current and $L_{1}$ is leakage inductance.

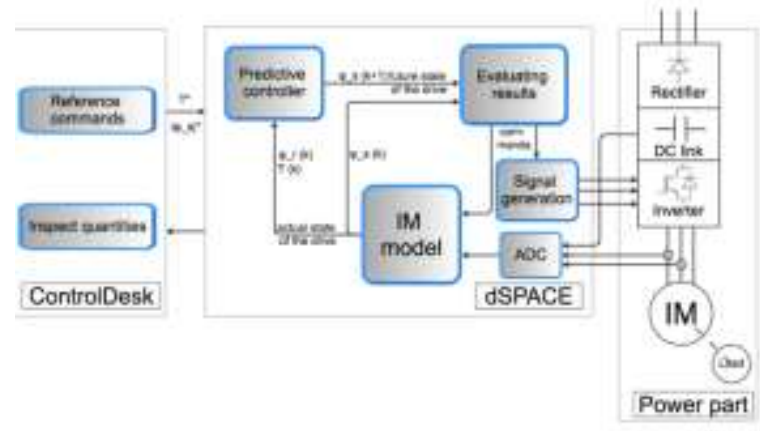

Fig. 1. Working principle. 
From the IM model the actual stator and rotor flux vectors and actual torque are known. The predictive controller calculates what the stator flux vector would look like in the very next step $\psi_{\mathrm{s}}(k+1)$. This flux vector can be defined as its amplitude and phase as (5):

$$
\overrightarrow{\Psi_{\mathrm{S}}(k+1)}=\left|\overrightarrow{\Psi_{\mathrm{S}}(k+1)}\right| \cdot e^{\mathrm{j} \theta_{\mathrm{S}}(k+1)}=\Psi_{\mathrm{S}}^{*} \cdot e^{\mathrm{j} \theta_{\mathrm{S}}(k+1)}
$$

where $k$ refers to the actual time sample, $k+1$ to the very next time sample, the upper script $*$ to the reference and $\theta_{\mathrm{s}}$ is the angle of the stator flux vector.

The amplitude of the flux vector changes slowly and during operation it is heading towards its reference. Because of that, it is possible to regard the reference amplitude as the next step value (5). The angle of the vector changes much more quickly and the correct determination of the angle is then important for the correct work of the method. The relations between flux vectors are depicted in Fig. 2.

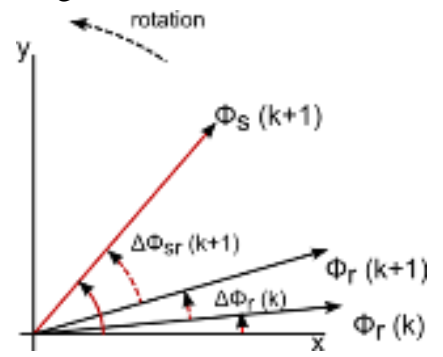

Fig. 2. Relations between magnetic fluxes.

The equation for the calculation of the desired angle is expressed as (6):

$$
\theta_{\mathrm{s}}(k+1)=\theta_{\mathrm{r}}(k)+\Delta \theta_{\mathrm{r}}(k)+\theta_{\mathrm{sr}}(k+1)
$$

where $\theta_{\mathrm{r}}$ is the angle of the rotor flux vector and $\Delta \theta_{\mathrm{r}}$ is the shift of the rotor flux vector angel during one step.

The first part of Eq. (6) is known from the mathematical model of IM as the rotor flux is calculated. From the transformation of Eq. (1), the angle between stator and rotor fluxes can be expressed as Eq. (7):

$$
\theta_{s r}(k)=\arcsin \left[-\frac{L_{\Delta} \cdot T(k)}{p_{p} \cdot L_{m} \cdot\left|\Psi_{s}(k)\right| \cdot\left|\Psi_{r}(k)\right|}\right]
$$

The algorithm requires the angle from Eq. (7) not for the actual sample but for the very next sample. Therefore, all time dependent variables must be replaced by their next sample. Because all of them cannot be calculated, some simplifications must be performed. Reaching the references of the stator flux vector amplitude and torque are expected to happen in the very next step. These variables can then be regarded as the references. Also the rotor time constant is much greater than the stator time constant and the changes performed in stator occur in rotor with certain delay. The change in amplitude of the rotor flux vector is then regarded to be zero and the amplitude for the next step can be replaced by the actual amplitude. This applies when the sampling time is adequately short. All of these steps are expressed in Eq. (8).

$$
\begin{aligned}
\theta_{\mathrm{sr}}(k+1) & =\arcsin \left[-\frac{L_{\Delta} \cdot T(k+1)}{p_{\mathrm{p}} \cdot L_{\mathrm{m}} \cdot\left|\Psi_{\mathrm{s}}(k+1)\right| \cdot\left|\Psi_{\mathrm{r}}(k+1)\right|}\right] \\
& =\arcsin \left[-\frac{L_{\Delta} \cdot T^{*}}{p_{\mathrm{p}} \cdot L_{\mathrm{m}} \cdot \Psi_{\mathrm{s}}^{*} \cdot\left|\Psi_{\mathrm{r}}(k)\right|}\right]
\end{aligned}
$$

In Eq. (6), the last unknown part is the shift of the rotor flux vector during one step. It can be expressed as a difference in Eq. (9).

$$
\frac{\mathrm{d} \theta_{\mathrm{sr}}}{\mathrm{dt}}=\frac{\mathrm{d}\left(\arctan \frac{\Psi_{\mathrm{r} \alpha}}{\Psi_{\mathrm{r} \beta}}\right)}{\mathrm{dt}}=\left(\frac{\mathrm{d} \Psi_{\mathrm{r} \alpha}}{\mathrm{d} t} \Psi_{\mathrm{r} \beta}-\frac{\mathrm{d} \Psi_{\mathrm{r} \alpha}}{\mathrm{d} t} \Psi_{\mathrm{r} \alpha}\right) /\left|\Psi_{\mathrm{r}}\right|^{2}
$$

Also, the rotor voltage equation of the induction motor can be transformed to express the derivation of the rotor flux.

$$
\frac{d \Psi_{r}}{d t}=j \cdot \omega \cdot \Psi_{r}-R_{r} \cdot i_{r}
$$

After discretization, the rotor voltage Eq. (10) can be substituted into Eq. (9) and the angle shift is then produced in Eq. (11):

$$
\Delta \theta_{\mathrm{r}}(k)=\left[\omega_{\mathrm{r}}(k)+\frac{R_{\mathrm{r}} \cdot T(k)}{p_{\mathrm{p}} \cdot\left|\Psi_{\mathrm{r}}(k)\right|^{2}}\right] \cdot T_{\mathrm{s}}
$$

where $R_{\mathrm{r}}$ is rotor resistance, $\omega_{\mathrm{r}}$ is electrical angular velocity of the rotor and $T_{\mathrm{s}}$ is sampling time.

Then the stator flux vector can be constructed from Eq. (5) as all parts of the equation are now calculated.

The inputs for the block evaluating results are the actual and the very next value of the stator flux vector. From these inputs, according to the stator voltage equation of the induction motor in Eq. (12) the voltage vector for the next step can be calculated. After discretization, the equation transforms to Eq. (13). Then the samples are moved forward by one step into Eq. (14).

$$
\begin{gathered}
\overrightarrow{u_{\mathrm{s}}}=R_{\mathrm{s}} \cdot \overrightarrow{u_{\mathrm{s}}}+\frac{\mathrm{d} \vec{\Psi}_{\mathrm{s}}}{\mathrm{d} t} \\
\overrightarrow{u_{\mathrm{s}}(k)}=\frac{\left[\overrightarrow{\Psi_{\mathrm{s}}(k)}-\overrightarrow{\Psi_{\mathrm{s}}(k-1)}\right]}{T_{\mathrm{s}}}+R_{\mathrm{s}} \cdot \overrightarrow{l_{\mathrm{s}}(k)} \\
\overrightarrow{u_{\mathrm{s}}(k+1)}=\frac{\left[\overrightarrow{\Psi_{\mathrm{s}}(k+1)}-\overrightarrow{\Psi_{\mathrm{s}}(k)}\right]}{T_{\mathrm{s}}}+R_{\mathrm{s}} \cdot \overrightarrow{l_{\mathrm{s}}(k)}
\end{gathered}
$$

The voltage vector $u_{\mathrm{s}}(k+1)$ is then the desired value that moves the drive towards the reference values. This method can then either use a modulation to create the necessary vector, put the nearest active combination of transistors for the whole step or put the zero vector combination to the inverter.

\section{IMPLEMENTATION}

At first, the described control strategy was examined in the MatLab Simulink environment. Then, it was tested on the real drive with the three phase induction motor of $5.5 \mathrm{~kW}$ nominal power and indirect frequency converter with the voltage type DC link. The nominal values of the motor are shown in Tab. I.

TABLE I.

INDUCTION MOTOR NOMINAL VALUES

\begin{tabular}{|l|l|l|}
\hline$U_{\mathrm{n}}$ & $230 \mathrm{~V}$ & Nominal voltage \\
\hline$I_{\mathrm{n}}$ & $11.8 \mathrm{~A}$ & Nominal current \\
\hline$P_{\mathrm{n}}$ & $5.5 \mathrm{~kW}$ & Nominal power \\
\hline$\omega_{\mathrm{n}}$ & $145 \mathrm{rad} / \mathrm{s}$ & Nominal speed \\
\hline & $\mathrm{Y}$ & Star connected \\
\hline$p_{\mathrm{p}}$ & 2 & Number of pole pairs \\
\hline
\end{tabular}




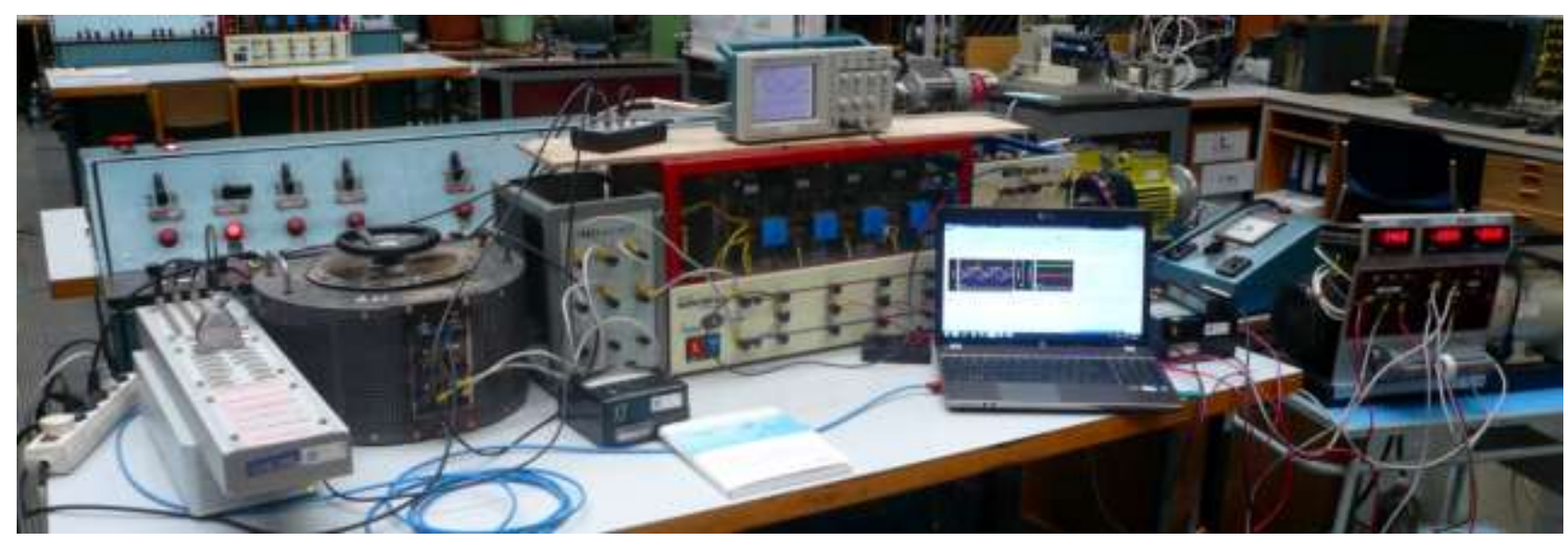

Fig. 3. Workplace.

The parameters of the motor equivalent circuit such as $R_{\mathrm{s}}, R_{\mathrm{r}}, L_{\mathrm{m}}$ or $L_{\Delta}$ were measured or calculated under specific conditions. The drive was then kept in these conditions during the whole measurement. This had to be performed in order to prevent the parameter from changing their values. The parameters of the equivalent circuit are shown in Tab. II.

TABLE II.

PARAMETERS OF EQUIVALENT CIRCUIT OF IM

\begin{tabular}{|l|l|l|}
\hline$R_{\mathrm{S}}$ & $0,952 \Omega$ & Stator resistance \\
\hline$L_{\mathrm{s} \sigma}$ & $0,0093 \mathrm{H}$ & Stator leakage inductance \\
\hline$R_{\mathrm{r}}$ & $0,952 \Omega$ & Rotor resistance \\
\hline$L_{\mathrm{r} \sigma}$ & $0,0072 \mathrm{H}$ & Rotor leakage inductance \\
\hline$L_{\mathrm{m}}$ & $0,129 \mathrm{H}$ & Magnetizing inductance \\
\hline
\end{tabular}

The load for the drive was created as a passive load by the separately excited DC motor. Its terminals were connected to the sliding resistance to adjust the load and the shaft was common with the tested induction motor. The currents flowing in two stator phases were measured using Hall's sensors and the current in the third phase was calculated. Also the voltage level in the DC link was measured and the voltage vector was reconstructed from this value and from the set combination of transistors in the inverter.

The practical implementation took place on the dSPACE DS1103 platform controlled through the connected computer. On the computer it was controlled by the ControlDesk program that is able to visualize program variables and record them online. The time loop of the control algorithm in the processor was set to $50 \mu \mathrm{s}$. This value also limited switching of the transistors in the inverter, so every change of the state of the transistor could be performed only once per $50 \mu \mathrm{s}$. In Fig. 4, a screenshot of the ControlDesk software is shown.

When the strategy was compared to the DTC method, the conditions for the DTC were set in a way, that it was the same as for the predictive method. The hysteresis levels of the torque and flux amplitude controllers were then set to their minimum values while maintaining the same number of switching. The references for both strategies were the torque and stator flux amplitudes.

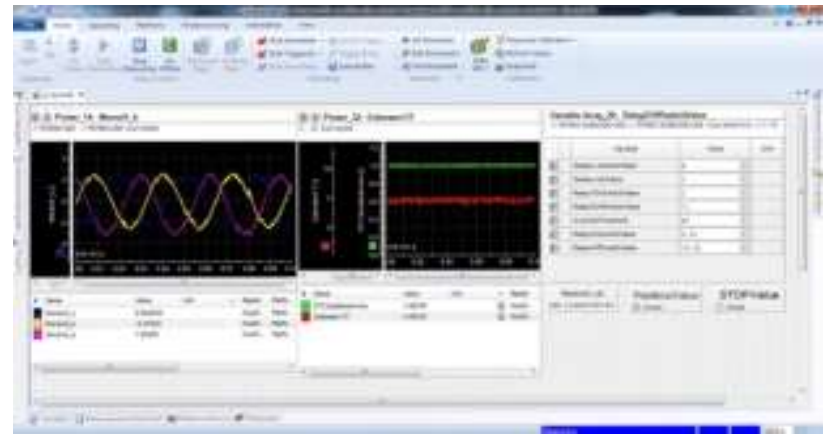

Fig. 4. ControlDesk.

\section{A. Simulation Results}

In the simulation environment the tests of the predictive method as well as of the DTC method were performed in order to compare the predictive method. The generated torque, stator flux amplitude, one phase of the stator current and switching of the transistors were recorded. The result of the performed simulation run is shown in Fig. 5 for torque, Fig. 6 for amplitude of the stator flux, Fig. 7 for the current and Fig. 8 for switching of the transistors. In Figs. 5 and 6, the lower range of the waveform ripples is visible in case of the predictive control, in Fig. 7, the distortion in the current waveform is lower in the predictive control, while the average switching of the transistors of the power inverter remained the same as it is evident in Fig. 8.

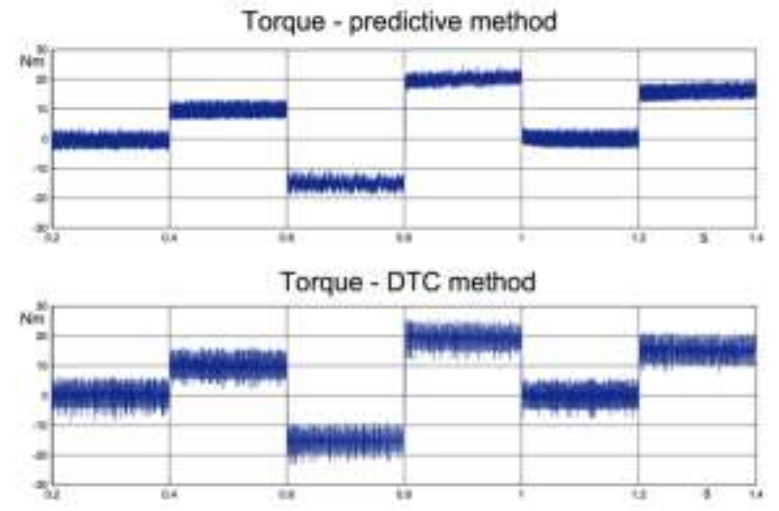

Fig. 5. Torque. 


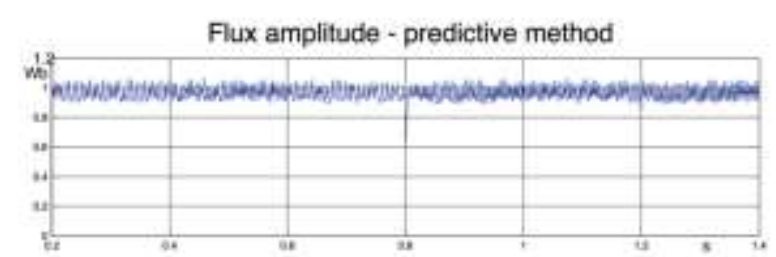

Flux amplitude - DTC method

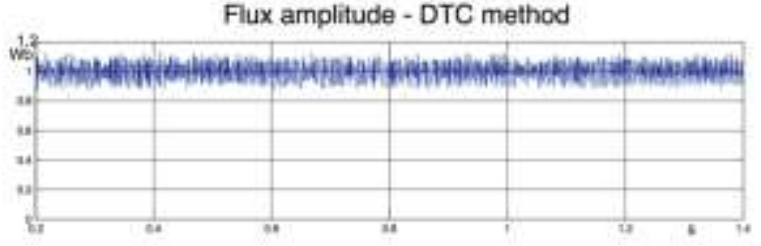

Fig. 6. Stator flux amplitude.
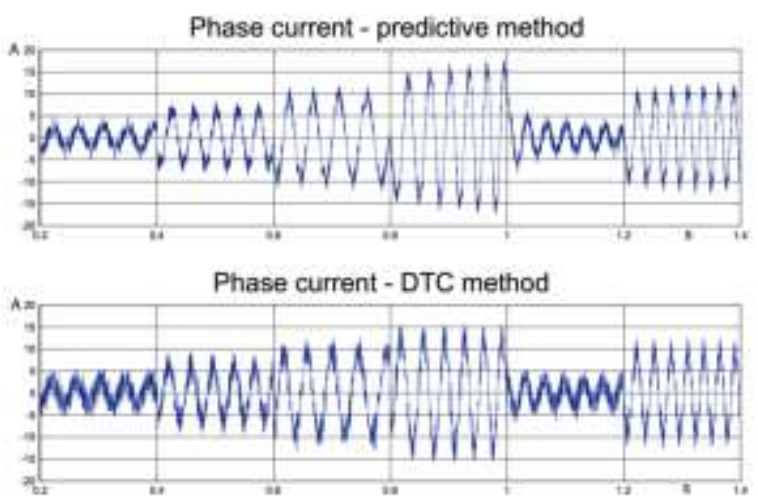

Fig. 7. Stator current.
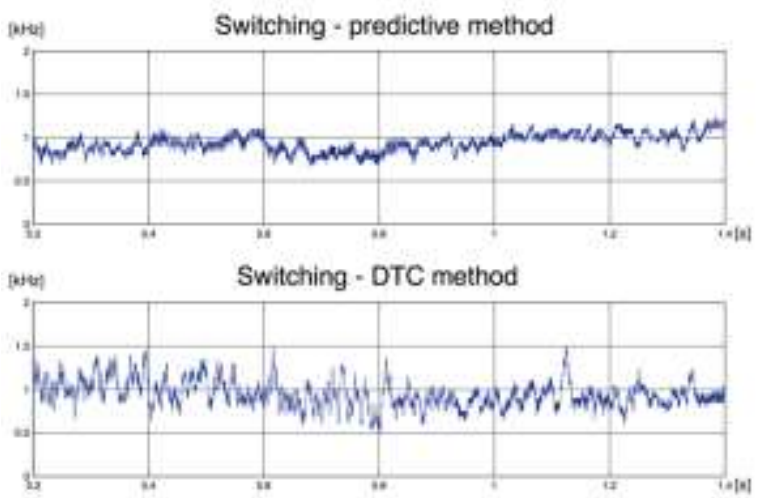

Fig. 8. Switching of the transistors.

\section{B. Experiment Results}

The control method was then implemented on the real drive with the induction motor and the behaviour was also examined. The trajectories of the stator flux are shown in Fig. 9 as an $\mathrm{X}-\mathrm{Y}$ graph, where the two components of the flux vector are displayed on individual axes. There can be seen that the tips of the both vectors are moving in a circular trajectory.
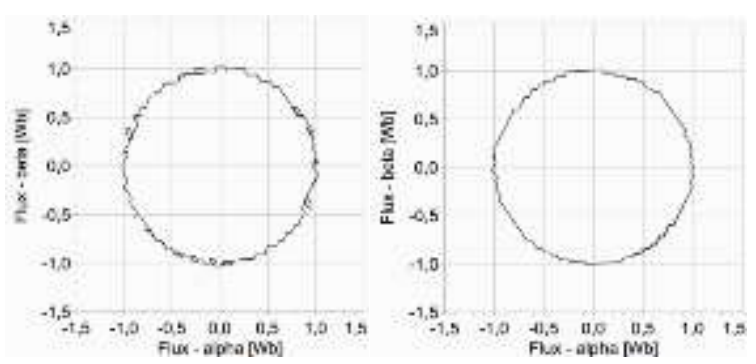

Fig. 9. Flux components shown in $\mathrm{X}-\mathrm{Y}$ graph; on the left - DTC, on the right - predictive method.

In Fig. 10, the actual and calculated flux vectors for the next step are presented. The red and blue curves are the predicted values and the black and green are the actual values.

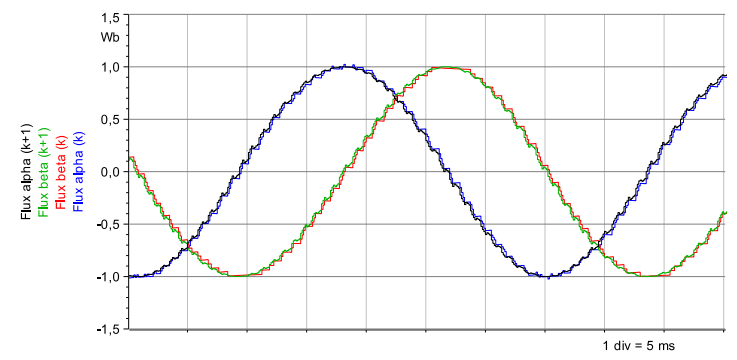

Fig. 10. Actual and predicted flux vector.

In Fig. 11, the torque, flux amplitude and currents are shown for the steady state.

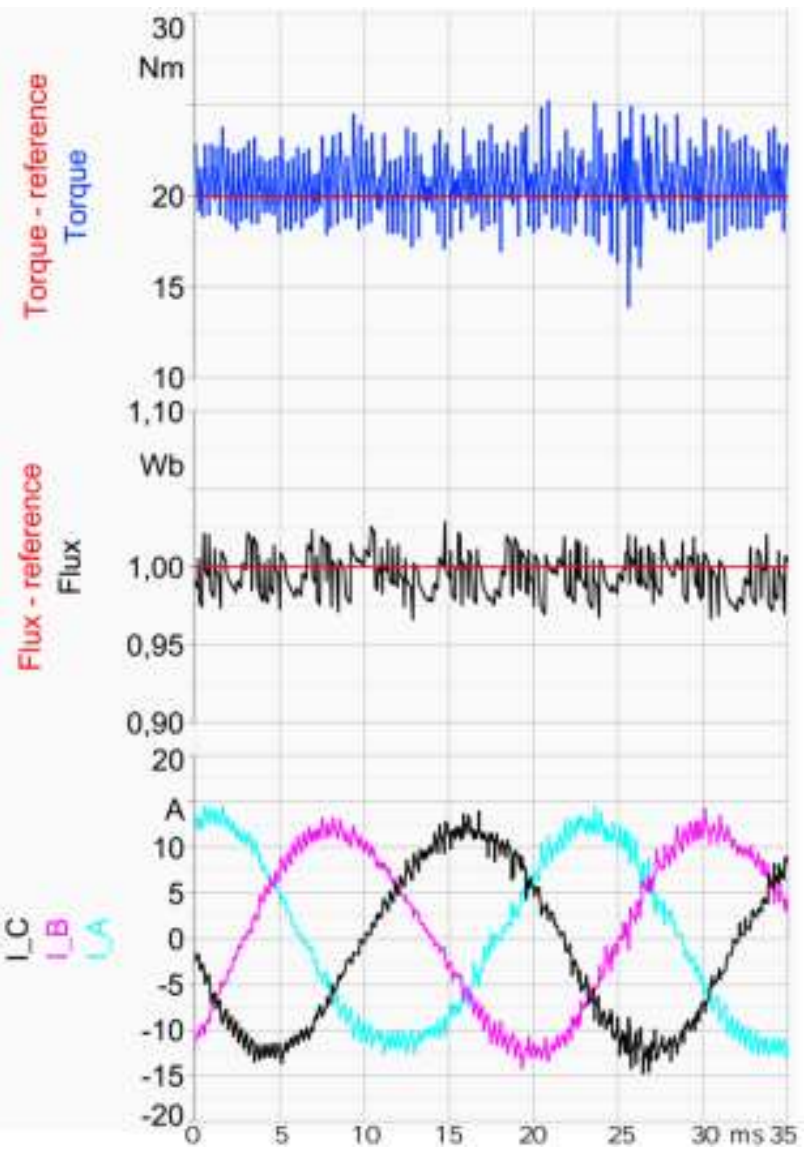

Fig. 11. Measured waveforms in steady state run. 


\section{CONCLUSION}

In the paper, a mathematical derivation of the suggested method of the predicted control of the drive with an induction motor is described. Then the implementation in the simulation environment and in experiment was performed and described. The results from the simulation and from measurement were presented in the paper together with its comparison to the classical DTC method.

The predictive method shows a good behaviour in steady state as well as in transients where it shows quick response to the step change in the torque reference. A stator flux vector tip is also maintained at a circular trajectory. When compared to the DTC method, a smother torque, flux and current waveforms are observable at the predictive method. The ripples in the torque waveform are reduced and the distortion in the flux amplitude and phase currents is lower while the number of switching of the transistors remains the same.

On the other side, the predictive method requires the knowledge of the rotor flux vector and therefore the more precise mathematical model of the induction motor must be included in the method. This mathematical model requires more parameters from the motor equivalent circuit and their change during time, temperature and magnetic feeding influence. The precision of the control and the waveforms become then smoother. In the laboratory measurement the drive was kept in condition when these parameters did not change. These conditions cannot be always ensured in real applications. If this condition is not fulfilled, some sophisticated methods for online identification of these parameters must be implemented to the control method. That is the same problem occurs for example in field oriented control and is often discussed. Other disadvantages of the predictive method is its time more consuming calculations and the necessity of the speed sensor.

\section{ACKNOWLEDGMENT}

This material is based on the work supported by the Technology Agency of the Czech Republic under the grant for Competence Centres Programme project No. TE02000103 and by the Student Grant Agency of Czech Technical University in Prague under grant No. SGS16/152/OHK3/2T/13.

\section{REFERENCES}

[1] I. Takahashi, T. Noguchi, "A new quick response and high efficiency control strategy of an induction motor", IEEE Trans. Ind. Appl., vol. IA-22, pp.820 -827 1986. https://doi.org/10.1109/TIA.1986.4504799

[2] K. B. Lee, F. Blaabjerg, and T. W. Yoon, "Speed-sensorless DTCSVM for matrix converter drives with simple nonlinearity compensation", IEEE Trans. Ind. Appl., vol. 43, no. 6, pp. 16391649, Nov./Dec. 2007 https://doi.org/10.1109/TIA.2007.908177

[3] B. S. Kumar, R. A. Gupta, R. Kumar, "12-Sector Methodology of Torque Ripple Reduction in a Direct Torque Controlled Induction Motor Drive", IEEE, SICE-ICASE, 2006. International Joint Conference, p. 3587 - 3592, Oct. 2006.

https://doi.org/10.1109/sice.2006.314746

[4] S. Kouro, R. Bernal, H. Miranda, C. A. Silva, and J. Rodriguez, "High performance torque and flux control for multilevel inverter fed induction motors," IEEE Trans. Power Electron., vol. 22, no. 6, pp., 2116-2123, Nov. 2007.

[5] Jef Beerten, Jan Verveckken, and Johan Driesen, "Predictive Direct Torque Control for Flux and Torque Ripple," IEEE Trans. Ind. Appl., vol. 57, no. 1, pp. 404-412, Jan. 2010. https://doi.org/10.1109/tie.2009.2033487

[6] Shengwen Fan, Jianxin Luo, Hu Zhang, A voltage vector prediction direct torque control system for induction motor, International Conference on Electrical and Control Engineering (ICECE), 2011. https://doi.org/10.1109/iceceng.2011.6058043

[7] P. Vas, "Sensorless vector and direct torque control", Oxford University Press, Oxford,1998.

[8] J. Rodriguez, P. Cortes, Predictive Control of Power Converteres and Electrical Drives, 246 pages, Wiley-IEEE Press, Apr. 2012. 